\title{
Rationale and Design of a Randomized, Double-Blind, Placebo Controlled Multicenter Trial to Study Efficacy, Security, and Long Term Effects of Intermittent Repeated Levosimendan Administration in Patients with Advanced Heart Failure: LAICA study
}

\author{
Martín J. García-González • Manuel de Mora-Martín • Silvia López-Fernández • \\ Javier López-Díaz • Manuel Martínez-Sellés • José Romero-García • Marco Cordero • \\ Antonio Lara-Padrón • Francisco Marrero-Rodríguez • M. del Mar García-Saiz • \\ Ana Aldea-Perona $\cdot$ on behalf of the LAICA study investigators
}

Published online: 27 July 2013

(C) The Author(s) 2013. This article is published with open access at Springerlink.com

\begin{abstract}
Background Advanced heart failure (HF) is associated with high morbidity and mortality; it represents a major burden for the health system. Episodes of acute decompensation requiring frequent and prolonged hospitalizations account for most HF-related expenditure. Inotropic drugs are frequently used during hospitalization, but rarely in outpatients. The LAICA clinical trial aims to evaluate the effectiveness and safety of monthly levosimendan infusion in patients with advanced HF to reduce the incidence of hospital admissions for acute HF decompensation.

Methods The LAICA study is a multicenter, prospective, randomized, double-blind, placebo-controlled, parallel group
\end{abstract}

A complete list of LAICA Investigators is shown in Appendix 1.

M. J. García-González $(\bowtie) \cdot M$. Cordero • A. Lara-Padrón •

F. Marrero-Rodríguez

Department of Cardiology, Hospital Universitario de Canarias,

Ctra. La Cuesta - Taco, Ofra s/n, 38320 San Cristóbal de La

Laguna, Sta. Cruz de Tenerife, Spain

e-mail: martinjgarciagonzalez@gmail.com

M. de Mora-Martín

Department of Cardiology, Hospital Regional Carlos Haya,

Málaga, Spain

S. López-Fernández

Department of Cardiology, Hospital Virgen de la Nieves, Granada,

Spain

J. López-Díaz

Department of Cardiology, Hospital Clínico Universitario de

Valladolid, Valladolid, Spain trial. It aims to recruit 213 out-patients, randomized to receive either a $24-\mathrm{h}$ infusion of levosimendan at $0.1 \mu \mathrm{g} / \mathrm{kg} / \mathrm{min}$ dose, without a loading dose, every 30 days, or placebo.

Results The main objective is to assess the incidence of admission for acute HF worsening during 12 months. Secondarily, the trial will assess the effect of intermittent levosimendan on other variables, including the time in days from randomization to first admission for acute HF worsening, mortality and serious adverse events.

Conclusions The LAICA trial results could allow confirmation of the usefulness of intermittent levosimendan infusion in reducing the rate of hospitalization for HF worsening in advanced HF outpatients.

\section{Martínez-Sellés}

Department of Cardiology, Hospital General Universitario

Gregorio Marañón, Universidad Europea, Madrid, Spain

J. Romero-García

Department of Cardiology, Fundación Jiménez Díaz, Madrid, Spain

M. del Mar García-Saiz

Department of Clinical Pharmacology, Hospital Universitario de Canarias, La Laguna, Sta. Cruz de Tenerife, Spain

\section{A. Aldea-Perona}

Clinical Trials Unit, Biomedical Research Unit, Fundación Rafael Clavijo para la Investigación Biomédica, Hospital Universitario de Canarias, La Laguna, Sta. Cruz de Tenerife, Spain 
Keywords Advanced heart failure $\cdot$ Levosimendan . Efficacy $\cdot$ Safety $\cdot$ Repeated administration $\cdot$ Outpatients

\section{Introduction}

Advanced heart failure (HF) is a health problem of increasing magnitude [1-3] despite the significant advances of recent decades [4]. It is characterized by repeated episodes of decompensation that cause frequent and prolonged hospitalizations [5]. As a consequence, the quality of life of these patients is severely compromised and the economic burden of healthcare is high $[6,7]$. Some patients may benefit from therapeutic measures with known impact on survival and quality of life, such as the implantation of circulatory assist devices and heart transplantation [8]. However, in many cases, such measures may be delayed due to shortage of donor organs, or contraindicated due to host factors such as advanced age and co-morbidity $[9,10]$.

In recent years a new drug, levosimendan, has emerged in the therapeutic management of HF. It is a calcium-sensitizing agent, which exerts a combined positive inotropic and vasodilator effect [11]. There is extensive scientific evidence on the effectiveness and utility of levosimendan in treating patients with acutely decompensated HF [12-15]. It is therefore commonly used in many hospitals around the world. However, there are no efficacy and safety data to support the use of intermittent levosimendan for the management of outpatients with advanced HF.

The LAICA study (ClinicalTrials.gov Identifier: NCT00988806/Number EudraCT: 2009-011441-11) is an independent multicenter clinical trial designed to evaluate the efficacy and safety of intermittent administration of levosimendan in continuous $24 \mathrm{~h}$ intravenous infusion as a treatment strategy to reduce the incidence of hospital admission for acutely decompensated HF, at 12 months follow-up, in patients with advanced HF.

\section{Methods}

Study Design, Duration, Levosimendan Administration and Treatments Allowed

The LAICA study is a multicenter, prospective, randomized, double-blind, placebo-controlled, parallel group trial. Candidate participants are patients with advanced HF [5], of any aetiology, with at least one episode of acute decompensation requiring admission within the last 6 months, and clinically stable at the time of inclusion in the study (see Table 1 for inclusion and exclusion criteria). Study duration is 12 months of treatment and 12 months of follow up. All patients receive standard treatment for HF, at the discretion of the physician
Table 1 Inclusion and exclusion criteria

$$
\begin{aligned}
& \text { Inclusion Criteria } \\
& \text { Age }>18 \text { years old } \\
& \text { Symptoms of heart failure such as dysnea or weakness on minimal } \\
& \text { physical activity or at rest (NYHA III-IV functional class) } \\
& \text { Congestive signs (jugular venous distension, liver distension, lung } \\
& \text { crepitations or rales, peripheral edema) o low-output signs at rest } \\
& \text { Evidence of severe heart dysfunction defined as one of the following : } \\
& \text { • Ejection fraction less than } 30 \% \\
& \text { • Diastolic dysfunction }>\text { grade III } \\
& \text { - Pulmonary capillary wedge pressure }>16 \text { mmHg and/or central } \\
& \text { venous pressure }>12 \mathrm{mmHg} \text { at pulmonary artery catheterization } \\
& \text { • NTproBNP levels }>3,000 \mathrm{ng} / \mathrm{mL} \text {, when non-cardiac conditions } \\
& \text { are ruled out }
\end{aligned}
$$

Severe impairment of functional capacity evidenced by one of the following:

- Six-minutes walking test in women and/or patients $>75$ years : $<300$ m response

- Oxygen consumption during cardiopulmonary treadmill test : $<12-14 \mathrm{~mL} / \mathrm{kg} / \mathrm{min}$ response

History of more than 1 hospitalization episode in the 6 months before randomization

Standard pharmacological treatment

\section{Exclusion criteria}

Allergy or hypersensitivity to levosimendan or any of the components used to prepare the drug sample

Creatinine clearance $<30 \mathrm{~mL} / \mathrm{min}$

Autoimmune disease

Pregnancy

Women of childbearing age

Cardiac conditions associated with cardiac filling or output obstruction Systolic tension $<90 \mathrm{mmHg}$

Heart rate $>120 \mathrm{bpm}$ or history of torsade de pointes

Treatment with vasoactive amines or phosphodiesterase inhibitors in the 2 weeks before randomization

Any associated disease with poor prognosis and/or reduced survival time Inability to give informed consent

Concomitant participation in other studies

responsible in each case, in accordance with current recommendations for the management of chronic heart failure [4] and may be carriers of an implantable cardioverter defibrillator or cardiac resynchronization therapy device. Patients are randomly assigned, in double-blind manner, to receive an infusion of levosimendan at $0.1 \mu \mathrm{g} / \mathrm{kg} / \mathrm{min}$, without a loading dose (Group I), or placebo of the same colour, at the same dosis (Group II), during $24 \mathrm{~h}$, once every 30 days. The dose is established on the basis of previous studies [14, 15], researcher experience and the usual practice in many centres. The absence of a loading dose is for safety reasons (to avoid the risk of episodes of hypotension). The infusion rate may be reduced to $0.05 \mu \mathrm{g} / \mathrm{kg} / \mathrm{min}$ or suspended, according to the patient's response to the drug (hypotension or tachycardia), 
in which case it will be recorded as a serious adverse event. Infusion of the drug will take place in a medical setting, under clinical supervision with electrocardiography and non-invasive hemodynamic monitoring. Clinical status of each participant will be evaluated at 15 days of infusion. If the patient is not found in a state of adequate compensation (defined as subjective improvement of HF symptoms and/or improvement by at least one NYHA functional class, and/or objective disappearance of signs of systemic venous congestion, pulmonary congestion and/or objective improvement in signs of peripheral perfusion) or if the clinical status worsens before the 30-day deadline for the next infusion, then the infusion timetable will be shortened to every 15 days with clinical assessments at 7 days. In cases of persistent decompensation after two consecutive infusions every 15 days, the randomization code will be disclosed and the patient receive the medical treatment deemed most appropriate by the attending physician, including heart transplantation when indicated. A complete summary of the protocol is depicted in Fig. 1.
Randomization, Infusion Masking Procedure and Ethical Considerations

Patients will be centrally randomized using a random number generator. Patient identification and drug/placebo numeric codes will be safeguarded in the Pharmacy Department, University Hospital of the Canary Islands. The infusion masking will only be removed in cases of a serious adverse event or when the primary endpoint of the trial is attained. The study drug and placebo will be presented as a concentrate in $5 \mathrm{ml}$ vials of identical appearance.

The study has been approved by local clinical research ethics committees of each participating center and by the health authorities (Spanish Agency of Medicines and Health Products, Ministry of Health). It will be conducted in accordance with the standards of Good Clinical Practice for trials with medical products in the European Community (http://www.ec.europa. eu/health/documents/eudralex/vol-10/index_en.htm) and the Declaration of Helsinki concerning medical research in human subjects [16]. An independent ad hoc clinical events committee, blinded to the treatment received, will supervise the course and

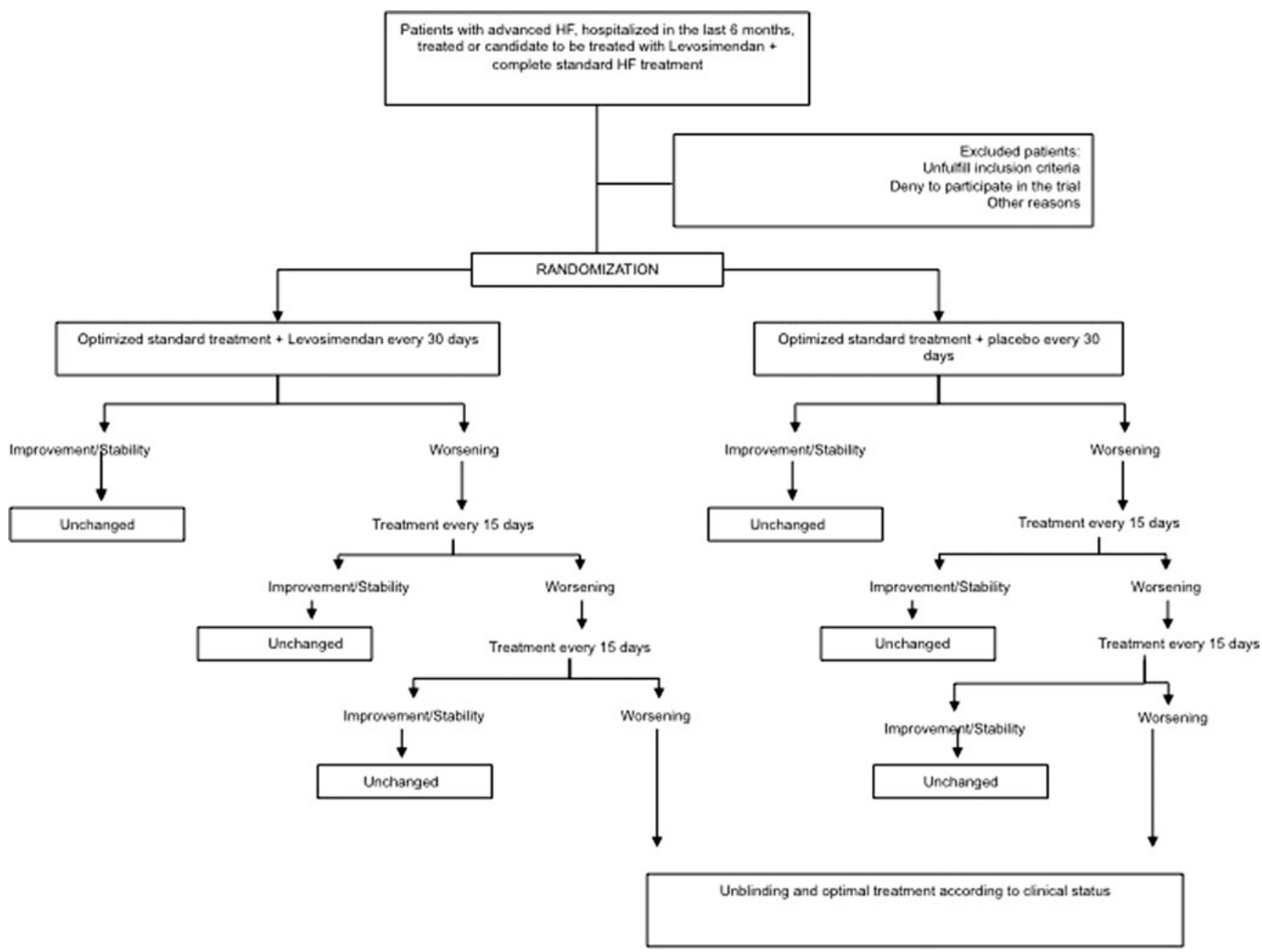

Fig. 1 Summary of study design protocol 
safety of the study. It will receive reports on all events relating to the primary objective, secondary objectives and adverse reactions that may occur during implementation. An interim analysis will be performed to evaluate the efficacy and safety of the study when the first 100 patients are recruited.

\section{Main and Secondary Objectives of the Study}

The primary endpoint of the trial is to determine the incidence of combined overall mortality and admission for acute HF decompensation or worsening. Clinical worsening or acutely decompensated heart failure is defined as admission to the emergency department or a hospital ward during more than $12 \mathrm{~h}$ due to worsening of symptoms and signs of HF. The effect of study drug administration on other secondary end-points will also be evaluated [Table 2].

The end-points of the study will be evaluated at patient visits between each administration of the study drug, based on history, physical signs, laboratory tests (blood count, renal function, ions and NTproBNP), echocardiography, functional assessment by treadmill test with measurement of myocardial oxygen consumption or a 6-min walking test, and evaluation of quality of life using the validated Spanish version of the Kansas City Cardiomyopathy Questionnaire [17] at baseline, and at 3,6 and 12 months of inclusion in the study.

Sub-Study on Renal Function Previous studies suggest that levosimendan treatment has a beneficial effect of on renal function [14]. The objective of this sub-study is to confirm any such effect on renal function in patients with advanced HF. Glomerular filtration rate will be estimated using the MDRD formula [18] after randomization, at 30 days, 3, 6 and 12 months of inclusion in the study. Additionally, cystatin $\mathrm{C}$ levels will be measured at baseline, and at 6 and 12 months of inclusion in the study.

Table 2 Primary and secondary end-points

Primary end-point
Combined overall mortality and admission for HF decompensation
or worsening
Secondary end-points
Time in days from randomization to first hospitalization for ADHF
Overall mortality at 1,6 and 12 months
Cardiac and non-cardiac mortality at 1,6,12 months
Cumulative incidence of mortality and hospitalization rate for ADHF
Incidence of Major Adverse Cardiac Events (MACE) such as sudden
death, myocardial ischemia, acute myocardial infarction
Time in days from randomization to any MACE
Changes in NYHA functional class from randomization to
$1,6,12$ months
NTproBNP changes before and after treatment
Quality of Life assessment at 1,6,12 months using KCCQ

Sub-Study on Cost-Effectiveness The objective of this substudy is to assess the impact in terms of cost-efficacy of repeated doses of levosimendan in patients with advanced HF. As markers of cost-effectiveness we will use the number of hospitalizations, number of days spent in the intensive care unit and/or hospital ward, and the number of visits to primary care. The impact on quality of life and health status of the participants will be assessed using the KCCQ questionnaire at baseline and at each follow-up visit.

Sample Size Rationale, Data Collection and Statistical Analysis In calculating the study sample size we used data from a previous study by W. Mullens et al. [19]: in a cohort of 513 advanced HF patients on standard treatment, the 1year incidence of hospital admission for HF worsening was $38 \%$. Thus, for our study to have a power of $80 \%$ and an alpha risk of 0.05 (one-tailed contrast) we need 213 patients (163 patients to receive levosimendan treatment and 50 patients placebo), assuming a final result of $18 \%$ reduction in the incidence of the primary endpoint of the study.

All patient data are to be collected centrally on an electronic data spreadsheet using a telematic application with predefined ranges, controls to prevent double entry and warnings about erroneous data entry. Patient identity will be encrypted to protect the safety and confidentiality of their data. Patients who during the study period are eligible but not randomized for any reason will be included in a simplified register and the reason for their non-inclusion will be specified, but they will be subject to follow-up at 30 days and 12 months.

Statistical analysis will be performed according to the intention-to-treat (ITT) principle. There will be a descriptive analysis of all variables collected (i.e. variables related to efficacy and safety, and all variables that may be considered as related to them) for both treatment groups. Variables will be compared between groups according to original patient randomization to a group (in accord with the ITT principle). Statistical analysis will be performed by an independent group of experts in biostatistics and research methodology. For missing data (partial and complete), statistical imputation with "last observation carried forward" will be used.

\section{Discussion}

The management of HF accounts for $2 \%$ of health expenditure in industrialized countries, and $70 \%$ of that is due to frequent and prolonged hospitalizations for acutely decompensated HF [6]. Strategies to reduce such hospitalizations could help reduce the burden of HF care. The aim of the LAICA study is to determine whether the intermittent administration of levosimendan by intravenous infusion during $24 \mathrm{~h}$ once every 30 days is safe and effective in reducing 
the incidence of admission for acute decompensation in patients with advanced HF.

The administration of inotropic drugs, by continuous or intermittent infusion, is frequently used in hospitalized patients with advanced HF in order to improve quality of life and survival or as a bridge to transplantation $[4,20]$. Although inotropes can immediately improve clinical status and hemodynamics, they may trigger and accelerate pathophysiological mechanisms that lead to increased myocardial damage. Numerous studies have shown that this method of administration has short-to-long term negative effects, including increased myocardial oxygen requirements, cardiotoxicity and pro-arrhythmogenic effects which significantly limit their usefulness as therapeutic agents [21,22].

Levosimendan, a calcium sensitizing agent, has inotropic and vasodilator effects exerted by a dual mechanism of action: 1) it increases the calcium sensitivity of troponin C, without increasing the release of calcium into the cytosol or modifying intracellular cyclic AMP levels, and 2) it activates $\mathrm{K}^{+}$-ATPasedependent channels and blocks the release of endothelin-1. This dual mechanism of action leads to improved myocardial contractility without either significant changes in oxygen requirements or pro-arrhythmic effects [11].

Different studies have demonstrated the effectiveness of levosimendan in treating acute HF decompensation from the clinical, hemodynamic, and even prognostic standpoint, compared with other treatment arms [12-15]. However, others have subsequently questioned the benefits for long-term prognosis of these patients $[23,24]$. This disparity of results has been attributed to methodological differences [25].

Preliminary studies, all with small sample sizes, have examined the effects of administering levosimendan in repeated doses for the treatment of HF. In 36 patients with advanced HF, Nanas et al. [26] studied the efficacy and safety of biweekly levosimendan associated with dobutamine compared with dobutamine alone. Patients receiving levosimendan survived longer.

Parisis et al. [27], in a randomized placebo-controlled study involving 25 patients with advanced HF, studied the effect of levosimendan infusion during $24 \mathrm{~h}$ every 3 weeks on cardiac function, geometry and levels of troponin T, NT-proBNP, high sensitivity C-reactive protein and interleukin-6. Repeated levosimendan infusions significantly improved geometry and cardiac function, and positively modulated neuro-hormonal activation and inflammatory response in patients with advanced $\mathrm{HF}$ without increasing myocardial damage.

Mavrogeni et al. [28], in 50 patients with advanced HF, prospectively evaluated the clinical effect of intermittent monthly infusion of levosimendan during $24 \mathrm{~h}$, compared to controls receiving standard care, for 6 months. The results showed significant symptom improvement in those receiving intermittent levosimendan, together with an increase in left ventricular systolic function.
Parle et al. [29], in a prospective observational study, studied the effectiveness of repeated infusions of levosimendan in 45 patients with advanced HF. They showed that levosimendan can be administered repeatedly, was well tolerated, with improvements in functional class and BNP levels after drug infusions.

Two current multicenter studies are aimed at assessing the efficacy and safety of intermittent levosimendan administration in patients with advanced HF. Altenberger et al. [30] in a prospective, randomized, double-blind, placebo-controlled, parallel group trial (the LevoRep study, ClinicalTrials.gov Identifier: NCT01065194) evaluated the efficacy and safety of levosimendan infusion every 2 weeks during $6 \mathrm{~h}$ in 120 outpatients with advanced HF. This study failed to show significant improvements in its primary objectives: to assess changes in functional capacity and quality of life at the end of the 24-week study period. However, the study meets its secondary end-points and showed that ambulatory levosimendan was safe and improves event-free survival by $50 \%$ in the short term ( 8 weeks) and long term ( 24 weeks) from randomization, compared with placebo. These authors agree that studies with more patients and higher dosing or higher repetition frequencies of levosimendan in an outpatient setting could show positive results for the primary endpoints [31].

The currently ongoing LION-HEART study (Clinical Trials.gov Identifier: NCT01536132), using a levosimendan administration regimen similar to that of the LevoRep study, aims to determine its efficacy and safety in 70 outpatients with advanced HF, compared with placebo, where the main outcome variable is the change in BNP levels from baseline to the end of a 3-month treatment period. Secondary objectives include the assessment of hospitalizations and mortality at 12 months follow up.

The design of our study differs substantially from these two studies in terms of the form and duration of drug administration, and the primary objective. However, we share the same intention of evaluating the efficacy and safety of intermittent levosimendan in patients with advanced HF. Results, if positive, may lead to recommendations based on scientific evidence in support of the strategy of repeated intermittent administration of levosimendan as an additional therapeutic step in the treatment of patients with advanced HF.

Lastly, the presence of renal function impairment or worsening complicates the management of patients with advanced $\mathrm{HF}$ and is an independent predictor of adverse outcome [32]. In the LIDO study, a significant reduction in serum creatinine levels was observed in levosimendantreated patients [14]. In addition, other clinical findings suggest that levosimendan treatment has a beneficial effect on renal function [32, 33]. The renal sub-study will allow us to detect this effect in patients with advanced HF. 


\section{Conclusions}

The LAICA study aims to assess the efficacy and safety of intermittent levosimendan administration as an additional therapeutic measure in patients with advanced HF, and to test the hypothesis that its use in outpatients could reduce the rate of admissions for acutely decompensated HF. If this is confirmed, it could have important implications for quality of life and healthcare costs associated with managing patients with advanced HF.

Acknowledgments All the authors wish to express their special thank to : Matti Kivikko, for advices and tips on study design; Alejandro Jimenez Sosa for his support, Orion Pharma for producing and providing gratuitely samples of drug and placebo.

Fundings Department of Pharmacy and Health Products. Ministry of Health, Social Services and Equality, Government of Spain 2009. (TRA-058).

Department of Health. Canary Islands Government. Fundación Canaria de Investigación y Salud (FUNCIS) 2010.

Additional research unrestricted grant from ORION PHARMA Laboratory.

Open Access This article is distributed under the terms of the Creative Commons Attribution License which permits any use, distribution, and reproduction in any medium, provided the original author(s) and the source are credited.

\section{Appendix 1}

\section{Executive Committee of LAICA Study}

Francisco Marrero-Rodríguez; Department of Cardiology. Hospital Universitario de Canarias. La Laguna. Sta. Cruz de Tenerife.

Martín J García-González; Department of Cardiology. Hospital Universitario de Canarias. La Laguna. Sta. Cruz de Tenerife.

Ana Aldea-Perona. Clinical Trials Unit (UCICEC) CAIBER. Unidad Mixta de Investigación. Fundación Rafael Clavijo para la Investigación Biomédica. Hospital Universitario de Canarias.

Emilia González Sola; Department of Cardiology. Hospital Universitario de Canarias. La Laguna. Sta. Cruz de Tenerife.

\section{Safety Committee of LAICA Study}

Ma del Mar García-Saiz. Department of Clinical Pharmacology Hospital Universitario de Canarias. La Laguna. Sta. Cruz de Tenerife.

Patricia Rodríguez-Fortúnez. Clinical Trials Unit (UCICEC) CAIBER. Unidad Mixta de Investigación. Fundación Rafael Clavijo para la Investigación Biomédica. Hospital Universitario de Canarias.
Geoffrey Yanes-Bowden. Department of Cardiology. Hospital Universitario de Canarias. La Laguna. Sta. Cruz de Tenerife.

Alejandro Sánchez-Grande. Department of Cardiology. Hospital Universitario de Canarias. La Laguna. Sta. Cruz de Tenerife.

Investigators and Participating Centers of the LAICA Study

Hospital Universitario de Canarias, La Laguna, Sta. Cruz de Tenerife,Spain: Martin J. García-González; Marco Cordero; Francisco Marrero-Rodríguez; Antonio Lara-Padrón, Antonio Barragán-Acea; Francisco Bosa-Ojeda; Juan LacalzadaAlmeida; Ignacio Laynez-Cerdeña; Tirso Virgos-Aller; Marina Bullejos-Molina; Inmaculada Rodríguez-Pedrosa. Hospital Regional Universitario Carlos Haya, Málaga, Spain: Manuel de Mora-Martín, Ana Mª González-González, Beatriz Pérez-Villardón.

Hospital Virgen De Las Nieves, Granada, Spain: Silvia López-Fernández, Inmaculada Zafra.

Hospital Clínico de Valladolid, Valladolid, Spain: Javier López-Díaz, Amada Recio, Carolina de Álvaro.

Hospital General Gregorio Marañón, Madrid, Spain:

Manuel Martínez-Sellés.

Fundación Jiménez Díaz, Madrid, Spain: José Romero-

García, Hans Paul Gaeper, Oscar González-Lorenzo

Hospital Sierrallana, Torrelavega, Santander, Spain: Pilar

Ortiz. Gonzalo Martín Gorria.

Hospital Santa María de Lleida, Lleida, Spain: Gerad

Torres-Cortada. Verónica Martínez-Fabra.

Hospital Universitari Arnau de Vilanova, Lleida, Spain: Jose Luis Morales-Rull.

\section{References}

1. Cleland JG, Khand A, Clark A. The heart failure epidemic: exactly how big is it? Eur Heart J. 2001;22:623-6.

2. Ceia F, Fonseca C, Mota T, Morais H, Matias F, de Sousa A, et al. Prevalence of chronic heart failure in Southwestern Europe: the EPICA study. Eur J Heart Fail. 2002;4:531-9.

3. Anguita Sánchez M, Crespo Leiro MG, De Teresa GE, Jiménez Navarro M, Alonso-Pulpón L, Muñiz García J, et al. Prevalence of heart failure in the Spanish general population aged over 45 years. The PRICE study. Rev Esp Cardiol. 2008;61:1041-9.

4. McMurray JJ, Adamopoulos S, Anker SD, Auricchio A, Böhm M, Dickstein $\mathrm{K}$, et al. ESC guidelines for the diagnosis and treatment of acute and chronic heart failure 2012: the Task Force for the Diagnosis and Treatment of Acute and Chronic Heart Failure 2012 of the European Society of Cardiology. Developed in collaboration with the Heart Failure Association (HFA) of the ESC. Eur J Heart Fail. 2012;14:803-69.

5. Metra M, Ponikowski P, Dickstein K, McMurray JJ, Gavazzi A, Bergh $\mathrm{CH}$, et al. Advanced chronic heart failure: a position statement from the Study Group on Advanced Heart Failure of the Heart 
Failure Association of the European Society of Cardiology. Eur J Heart Fail. 2007;9:684-94.

6. Stewart S, Jenkins A, Buchan S, McGuire A, Capewell S, McMurray JJ. The current cost of heart failure to the National Health Service in the UK. Eur J Heart Fail. 2002;4:361-71.

7. Hauptman PJ, Swindle J, Burroughs TE, Schnitzler MA. Resource utilization in patients hospitalized with heart failure: insights from a contemporary national hospital database. Am Heart J. 2008;155:978-85.

8. Slaughter MS, Singh R. The role of ventricular assist devices in advanced heart failure. Rev Esp Cardiol. 2012;65:982-5.

9. Mehra MR, Kobashigawa J, Starling R, Russell S, Uber PA, Parameshwar J, et al. Listing criteria for heart transplantation: International Society for Heart and Lung Transplantation guidelines for the care of cardiac transplant candidates - 2006. J Heart Lung Transplant. 2006;25:1024-42.

10. De Mora-Martín M, Pérez-Ruiz JM, Delgado-Prieto JL, UrbanoCarrillo CA. Comorbidity in patients admitted to a department of cardiology due to heart failure. Rev Esp Cardiol. 2011;64:75-83.

11. Innes CA, Wagstaff AJ. Levosimendan: a review of its use in the management of acute decompensated heart failure. Drugs. 2003;63:2651-71.

12. Nieminen MS, Akkila J, Hasenfuss G, Kleber FX, Lehtonen LA, Mitrovic V, et al. Hemodynamic and neurohumoral effects of continuous infusion of levosimendan in patients with congestive heart failure. J Am Coll Cardiol. 2000;36:1903-12.

13. Kivikko M, Lehtonen L, Colucci WS. Sustained hemodynamic effects of intravenous levosimendan. Circulation. 2003;107:81-6.

14. Follath F, Cleland JG, Just H, Papp JG, Scholz H, Peuhkurinen K, et al. Efficacy and safety of intravenous levosimendan compared with dobutamine in severe low-output heart failure (the LIDO study): a randomised double-blind trial. Lancet. 2002;360:196-202.

15. Moiseyev VS, Põder P, Andrejevs N, Ruda MY, Golikov AP, Lazebnik LB, et al. Safety and efficacy of a novel calcium sensitizer, levosimendan, in patients with left ventricular failure due to an acute myocardial infarction. A randomized, placebo-controlled, double-blind study (RUSSLAN). Eur Heart J. 2002;23:1422-32.

16. http://www.wma.net/es/30publications/10policies/b3/ DoH Oct2008

17. Green CP, Porter CB, Bresnahan DR, Spertus JA. Development and evaluation of the Kansas City Cardiomyopathy Questionnaire: a new health status measure for heart failure. J Am Coll Cardiol. 2000;35:1245-55.

18. O'Meara E, Chong KS, Gardner RS, Jardine AG, Neilly JB, McDonagh TA. The Modification of Diet in Renal Disease (MDRD) equations provide valid estimations of glomerular filtration rates in patients with advanced heart failure. Eur J Heart Fail. 2006;8:63-7.

19. Mullens W, Abrahams Z, Skouri HN, Taylor DO, Starling RC, Francis GS, et al. Prognostic evaluation of ambulatory patients with advanced heart failure. Am J Cardiol. 2008;101:1297-302.

20. Bayram M, De Luca L, Massie MB, Gheorghiade M. Reassessment of dobutamine, dopamine, and milrinone in the management of acute heart failure syndromes. Am J Cardiol. 2005;96:G47-58.
21. Felker GM, O'Connor CM. Inotropic therapy for heart failure: an evidence-based approach. Am Heart J. 2001;142:393-401.

22. Teerlink JR, Metra M, Zacà V, Sabbah HN, Cotter G, Gheorghiade $\mathrm{M}$, et al. Agents with inotropic properties for the management of acute heart failure syndromes. Traditional agents and beyond. Heart Fail Rev. 2009;14:243-53.

23. Packer M. REVIVE II: multicenter placebo controlled trial of levosimendan on clinical status in acutely decompensated heart failure. American Heart Association Scientific Sessions 2005, November 13-16, Dallas, Texas. Late breaking clinical trials II. Circulation. 2005;112:3363.

24. Mebazaa A, Nieminen MS, Packer M, Cohen-Solal A, Kleber FX, Pocock SJ, et al. Levosimendan vs dobutamine for patients with acute decompensated heart failure. The SURVIVE randomised trial. JAMA. 2007;297:1883-91.

25. Smetana P, Geppert A. Levosimendan in cardiogenic shock: the magic drug for every patient? Crit Care Med. 2007;35:2862-4.

26. Nanas JN, Papazoglou P, Tsagalou EP, Ntalianis A, Tsolakis E, Terrovitis JV, et al. Efficacy and safety of intermittent, long-term, concomitant dobutamine and levosimendan infusions in severe heart failure refractory to dobutamine alone. Am J Cardiol. 2005;95:768-71.

27. Parissis JT, Adamopoulos S, Farmakis D, Filippatos G, Paraskevaidis I, Panou F, et al. Effects of serial levosimendan infusions on left ventricular performance and plasma biomarkers of myocardial injury and neurohormonal and immune activation in patients with advanced heart failure. Heart. 2006;92:1768-72.

28. Mavrogeni S, Giamouzis G, Papadopoulou E, Thomopoulou S, Dritsas A, Athanasopoulos G, et al. A 6-month follow-up of intermittent levosimendan administration effect on systolic function, specific activity questionnaire, and arrhythmia in advanced heart failure. J Card Fail. 2007;13:556-9.

29. Parle NM, Thomas MD, Dembo L, Best M, Driscoll GO. Repeated infusions of levosimendan: well tolerated and improves functional capacity in decompensated heart failure - a single-centre experience. Heart Lung Circ. 2008;17:206-10.

30. Altenberger J, Parissis JT, Ulmer H, Poelzl G, LevoRep Investigators. Rationale and design of the multicentre randomized trial investigating the efficacy and safety of pulsed infusions of levosimendan in outpatients with advanced heart failure (LevoRep study). Eur J Heart Fail. 2010;12:186-92.

31. G. Poelzl. Efficacy and safety of intermittent ambulatory infusions of Levosimendan in end-stage heart failure (LevoRep Study) - Late breaking trial session - HEART FAILURE 2013. http://www. escardio.org/about/press/press-releases/pr-13/Pages/heart-failure-2013late-breaking-trials-2.aspx. - Accessed 19.06.2013.

32. Heywood JT, Fonarow GC, Costanzo MR, Mathur VS, Wigneswaran JR, Wynne J. High prevalence of renal dysfunction and its impact on outcome in 118,465 patients hospitalized with acute decompensated heart failure: a report from the ADHERE database. J Card Fail. 2007;13:422-30.

33. Zemljic G, Bunc M, Yazdanbakhsh AP, Vrtovec B. Levosimendan improves renal function in patients with advanced heart failure awaiting cardiac transplantation. J Card Fail. 2007;13:417-21. 